\title{
The clinical significance of intrahepatic Th22 cells in liver cirrhosis
}

\author{
Shanyu Qin ${ }^{A, D, F}$, Mei Chen ${ }^{B, C}$, Xiaoyun Guo ${ }^{B, F}$, Wei Luo ${ }^{C, D, F}$, Jiaxu Wang ${ }^{B, C, F}$, Haixing Jiang ${ }^{A, E, F}$ \\ Department of Gastroenterology, First Affiliated Hospital of Guangxi Medical University, Nanning, China \\ A - research concept and design; $B$ - collection and/or assembly of data; $C$ - data analysis and interpretation; \\ $D$ - writing the article; $E$ - critical revision of the article; $F$ - final approval of the article
}

\section{Address for correspondence}

Haixing Jiang

E-mail: gxjianghx@163.com

Funding sources

None declared

Conflict of interest

None declared

Received on November 23, 2015

Reviewed on December 30, 2015

Accepted on August 8, 2018

Published online on February 8, 2019

Cite as

Qin S, Chen M, Guo X, Luo W, Wang J, Jiang H. The clinical

significance of intrahepatic Th22 cells in liver cirrhosis.

Adv Clin Exp Med. 2019;28(6):765-770. doi:10.17219/

acem/94062

DOI

10.17219/acem/94062

\section{Copyright}

Copyright by Author(s)

This is an article distributed under the terms of the

Creative Commons Attribution Non-Commercial License

(http://creativecommons.org/licenses/by-nc-nd/4.0/)

\begin{abstract}
Background. Th22 cells are a recently identified $\mathrm{CD}^{+}{ }^{+}$Thelper subset and have been implicated in the pathogenesis of certain diseases in humans, but the role of Th22 cells in liver cirrhosis (LC) remains unclear.

Objectives. The aim of the study was to investigate the expression and clinical significance of intrahepatic Th22 cells in LC tissues.

Material and methods. Samples of liver tissue of $20 \mathrm{LC}$ patients and 12 normal controls (NC) were collected. Interleukin 22 (IL-22), IL-22R1 mRNA and aryl hydrocarbon receptor (AHR) expression were examined using quantitative reverse transcription polymerase chain reaction (RT-PCR). The protein expression of Th22 and $\mathrm{CD4}^{+}$cells in liver tissue was measured with immunohistochemistry.

Results. The number of intrahepatic Th22 and $\mathrm{CD}^{+}{ }^{+}$cells increased markedly in $\mathrm{LC}$ patients and the number of Th22 cells positively correlated with the number of $\mathrm{CD}^{+}{ }^{+}$cells $(p<0.05)$. Moreover, the number of Th22 cells positively correlated with the serum levels of alanine aminotransferase (ALT) and aspartate aminotransferase (AST), as well as the Child-Pugh score in LC patients $(p<0.05)$. The expression of $I L-22, I L-22 R 1$ and AHR in LC patients was significantly increased compared with the NC group $(p<0.05)$.
\end{abstract}

Conclusions. Our findings suggest that the expression of intrahepatic Th22 cells increased in LC patients and was associated with the progression of $\mathrm{LC}$.

Key words: Th22 cells, liver cirrhosis, clinical significance 


\section{Introduction}

Chronic hepatitis B (CHB) infection affects approx. 350 million individuals worldwide. It has become a major public health threat, especially in China, where 0.5-1.0 million individuals die due to $\mathrm{HBV}$-associated liver diseases each year. ${ }^{1}$ Liver cirrhosis (LC) is defined as the accumulation of extracellular matrix (ECM) forming fibrous scars that distort the hepatic architecture, and the subsequent development of nodules of regenerating hepatocytes.

It has been demonstrated that $\mathrm{CD}^{+}$cells are essential to controlling HBV infection. ${ }^{2} \mathrm{Th} 22$ cells are a recently identified CD4 ${ }^{+} \mathrm{T}$ helper subset distinct from Th17 and Th1 cells. They are characterized by particularly high interleukin 22 (IL-22) production, but not IL-17 or interferon gamma (IFN- $\gamma$ ), and they express the chemokine receptors CCR4, CCR6 and CCR10. ${ }^{3,4}$ Th22 cells have been implicated in the pathogenesis of certain diseases in humans, such as psoriasis, ${ }^{5}$ Crohn's disease $^{6}$ and gastric cancer. ${ }^{7}$ However, the nature of Th 22 cells in $\mathrm{HBV}$-associated LC remains poorly understood.

In the present study, we investigated the number of Th22 and $\mathrm{CD}^{+}$cells in the liver tissue of patients with HBVassociated $\mathrm{LC}$ and analyzed possible associations between Th2 2 and CD4 ${ }^{+}$cells and various clinical parameters, as well as the expression of transcription factor aryl hydrocarbon receptor (AHR), IL-22 and IL-22R1, aiming to reveal the possible mechanism of Th22 cell involvement in $\mathrm{HBV}$-associated liver cirrhosis.

\section{Material and methods}

\section{Patient selection}

Between October 2012 and May 2014, liver biopsies or surgically removed specimens were collected from $20 \mathrm{HBV}$ LC patients; samples from 12 patients who had undergone hepatic hemangiomas or cysts and had normal liver tissues were used as normal controls (NC). The diagnoses of LC were made according to previously described criteria. ${ }^{8,9} \mathrm{~Pa}$ tients who were co-infected with human immunodeficiency virus (HIV) or other hepatitis viruses or autoimmune diseases were excluded from the study group. None of the participants received anti-HBV agents or steroids within 6 months prior to the sampling. Patients with viral hepatitis, autoimmune hepatitis and alcoholic liver diseases were excluded from the NC group. Written informed consent was obtained from each participant and the study protocol was approved by the Ethics Committee of the First Affiliated Hospital of Guangxi Medical University, Nanning, China.

\section{Real-time polymerase chain reaction analysis}

The total RNA of the liver samples was isolated from the liver tissues using Invitrogen TRIzol Reagent (Thermo
Fisher Scientific Inc., Waltham, USA). cDNA synthesis was performed using PrimeScript RT Reagent Kits (Roche Diagnostics Corp., Indianapolis, USA). Real-time polymerase chain reaction (RT-PCR) was performed using Real-Time PCR Master Mix (Roche Diagnostics) according to the manufacturer's protocol. The primers are described as follows: IL-22 forward: ACT GGA TTT GCT GTT TAT GTC TCT G; IL-22 reverse: GGC TTC CCA TCT TCC TTT TG; IL-22R1 forward: TGG CAA AGA AGG GCT GTC AG; IL-22R1 reverse: GCG GTG ACC CTG GCA TAG T; AHR forward: TGT CGT CTA AGG TGT CTG CTG GA; AHR reverse: ACA AAG CCA ACT GAG GTG GAA GTA; GAPDH forward: GCA CCG TCA AGG CTG AGA AC; GAPDH reverse: TGG TGA AGA CGC CAG TGG A. GAPDH was measured as a housekeeping gene. Real-time PCR was performed using SYBR Green PCR Master Mix (Roche Diagnostics) on an ABI 7500 Real-Time PCR System (Applied Biosystems, Thermo Fisher Scientific). After an initial denaturation step for $3 \mathrm{~min}$ at $94^{\circ} \mathrm{C}$, a 3-step cycling procedure (denaturation at $94^{\circ} \mathrm{C}$ for $30 \mathrm{~s}$, annealing at $60^{\circ} \mathrm{C}$ for $30 \mathrm{~s}$ and extension at $72^{\circ} \mathrm{C}$ for $60 \mathrm{~s}$ ) was used for 35 cycles. Samples were assayed in triplicate. The expression ratio of each gene of interest (GOI) between different groups was analyzed using the $\Delta \Delta \mathrm{CT}$ method.

\section{Immunohistochemistry}

Immunohistochemical staining was performed with the streptavidin-biotin complex method, using streptavidin and biotin (Thermo Fisher Scientific), according to the manufacturer's instructions. Paraffin-embedded, formalin-fixed liver tissues were cut into $3-\mu \mathrm{m}$ sections and placed on polylysine-coated slides. Each paraffin section was deparaffinized and rehydrated through a graded series of ethanol. Endogenous peroxidase was blocked using a $3 \% \mathrm{H}_{2} \mathrm{O}_{2}$ methanol solution, and $5 \%$ goat serum albumin (Zhongshan Golden Bridge Biotech, Beijing, China) was applied to block non-specific staining. A 1:500 dilution of rabbit anti-human IL-22 (Thermo Fisher Scientific) and ready-to-use rabbit anti-human CD4 antibody (Zhongshan Golden Bridge Biotech) were used for the immunohistochemistry staining of IL-22 and CD4. Incubation of a biotin-free secondary antibody and horseradish peroxidase (Zhongshan Golden Bridge Biotech) was subsequently performed, followed by development with diaminobenzidine (Zhongshan Golden Bridge Biotech) and counterstaining with hematoxylin. Instead of a primary antibody, non-immune goat serum was taken as a control. The rates of positive staining in 5 randomly chosen high-power $(\times 400)$ fields of view were counted separately by 2 experienced pathologists. The numbers of positive cells in the same 5 high-power fields of view were also counted. The mean frequency of positive staining in each slide was then obtained. None of the pathologists were aware of the clinical diagnosis of the patient associated with any tissue section at the time of analysis. 


\section{Statistical analysis}

All the data was analyzed using SPSS software v. 16.0 for Windows (SPSS Inc., Chicago, USA). The results are presented as means with standard deviation (SD) using GraphPad Prism 5 software (GraphPad Software Inc., La Jolla, USA). Demographic and clinical data between groups was compared using the $\chi^{2}$ test or Student's t-test, as appropriate. Pearson's correlation coefficient or Spearman's rank correlation coefficient were used for correlation analysis depending on the data distribution. A value of $\mathrm{p}<0.05$ was considered significant.

\section{Results}

\section{Clinical data of the subjects}

Table 1 shows the clinical data of the LC and NC groups. There were statistically significant differences

Table 1. Clinical characteristics of the study participants

\begin{tabular}{|l|c|c|c|}
\multicolumn{1}{|c|}{ Parameters } & LC group & NC group & p-value \\
\hline Cases, $n$ & 20 & 12 & - \\
\hline Gender, $n$ (male/female) & $16 / 4$ & $7 / 5$ & 0.240 \\
\hline Age [years] & $42(13.75)$ & $44.5(12.5)$ & 0.421 \\
\hline ALT serum level [U/L] & $32(21.75)$ & $22.5(16.25)$ & 0.025 \\
\hline AST serum level $[\mathrm{U} / \mathrm{L}]$ & $26(15.75)$ & $20.5(10.75)$ & 0.015 \\
\hline TBIL serum level $[\mu \mathrm{mol} / \mathrm{L}]$ & $20.45(13.83)$ & $10.55(6.93)$ & 0.000 \\
\hline HBeAg $(+/-)$ & $7 / 13$ & $0 / 12$ & 0.029 \\
\hline HBV DNA (+/-) & $11 / 9$ & $\mathrm{ND}$ & - \\
\hline Child-Pugh score (A/B/C) & $12 / 8 / 0$ & $12 / 0 / 0$ & 0.014 \\
\hline
\end{tabular}

LC group - HBV-associated liver cirrhosis patients; NC group - normal controls; ALT - alanine aminotransferase; AST - aspartate aminotransferase; TBIL - total bilirubin; HBeAg - hepatitis Be antigen; ND - not determined; The data for age group, ALT, AST, and TBIL are shown as medians (interquartile range)
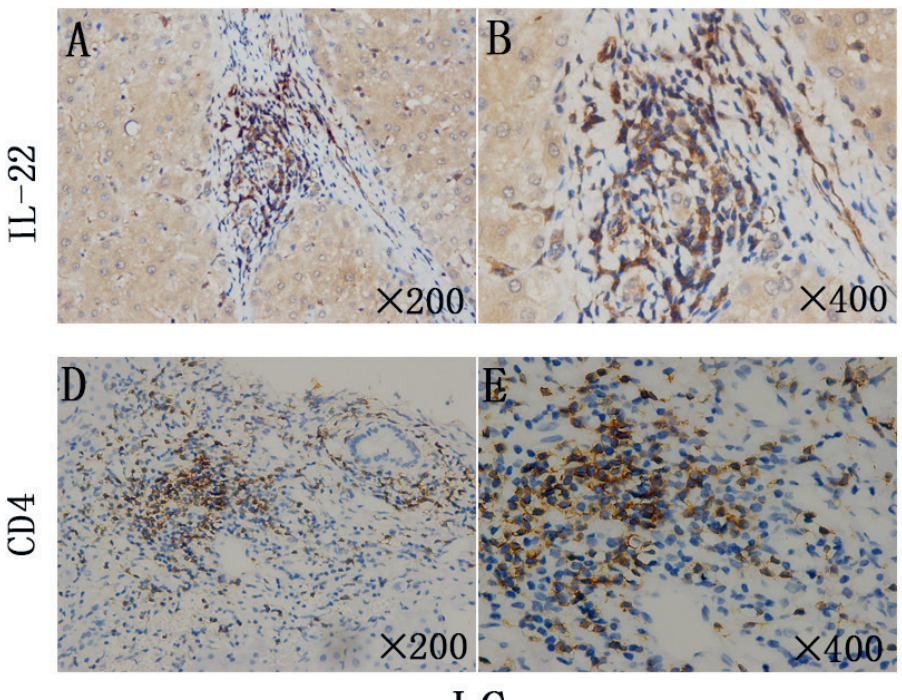

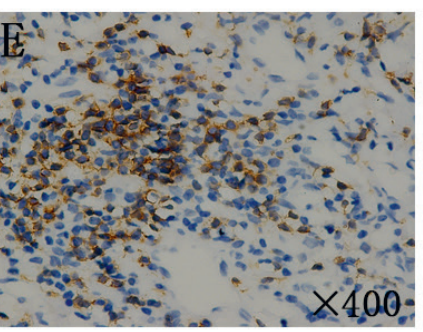

C in the serum concentrations of alanine aminotransferase (ALT0), aspartate aminotransferase (AST), total bilirubin (TBIL), the hepatitis Be antigen ( $\mathrm{HBeAg}$ ) positive rates, and Child-Pugh scores between the LC and NC patients ( $\mathrm{p}<0.05)$. No significant differences were found between the LC and NC patients in terms of gender or age ( $p>0.05)$.

\section{Distribution of Th22 and CD4+ cells in liver tissues}

Immunohistochemistry showed that IL-22 had a cytoplasmic staining pattern, and a large number of lymphocytes had infiltrated the livers of patients with HBV-associated LC (Fig. 1A,B). Th22 cells were mainly located in the portal area of the liver and could also be found in the lobes, especially in the inflammatory regions (Fig. 1B). In contrast, few or no Th22 cells were observed in the NC group (Fig. 1C). The CD4+T cells had mainly infiltrated the portal areas of the liver in the LC group, and were distinctly expressed in the membranes of the cells (Fig. 1D,E), but were rarely found in the NC group (Fig. 1F).

A significantly stronger expression of Th22 cells was detected in the LC patients $(65.06 \pm 35.01)$ than in the $\mathrm{NC}$ group $(4.17 \pm 2.79, \mathrm{p}<0.001$, Fig. $2 \mathrm{~A})$. The frequencies of $C D 4^{+} \mathrm{T}$ cells were significantly increased in the LC group $(70.94 \pm 25.13)$ compared with the NC group (4.5 \pm 1.76 , $\mathrm{p}<0.001$, Fig. 2B). According to the immunohistochemistry results, the number of Th22 cells in the liver was positively correlated with the number of $\mathrm{CD} 4{ }^{+} \mathrm{T}$ cells in both the NC group ( $r=0.944, p=0.005$; Fig. $2 \mathrm{C}$ ) and the LC group $(\mathrm{r}=0.966, \mathrm{p}<0.01$; Fig. 2D).

\section{Correlation between the number of Th22 cells and clinical parameters}

In the LC patients, the number of Th22 cells was positively correlated with the serum levels of ALT $(r=0.642$,
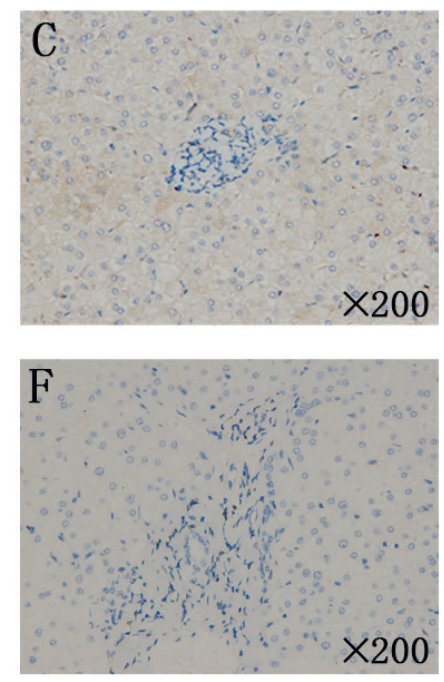

$\mathrm{NC}$
Fig. 1. Liver infiltration of Th22 and $C D 4^{+} T$ cells

Representative distribution of IL-22 and CD4 positive cells in liver samples of liver cirrhosis $(\mathrm{LC})$ patients (A, B, D, E) and normal controls (NC) (C, F). Positive staining is shown as a claybank color $(\times 200)$. The micrographs at higher magnification $(\times 400)$ show IL-22 distinctly expressed in the cytoplasm of the lymphocytes (B) while CD4 show membrane staining (E). 
A

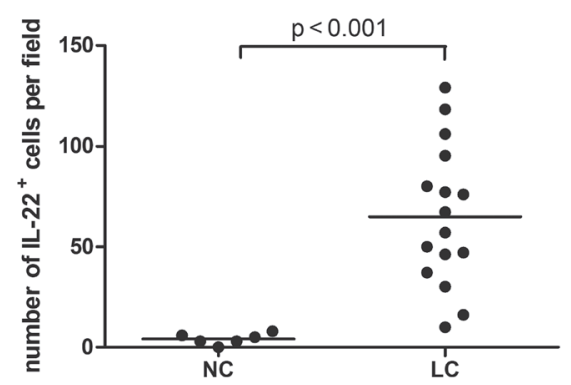

C

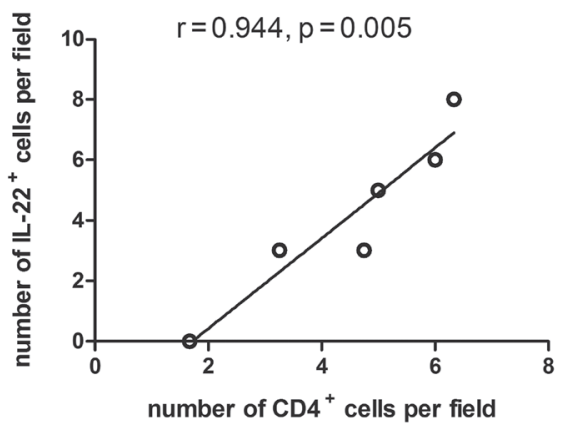

A

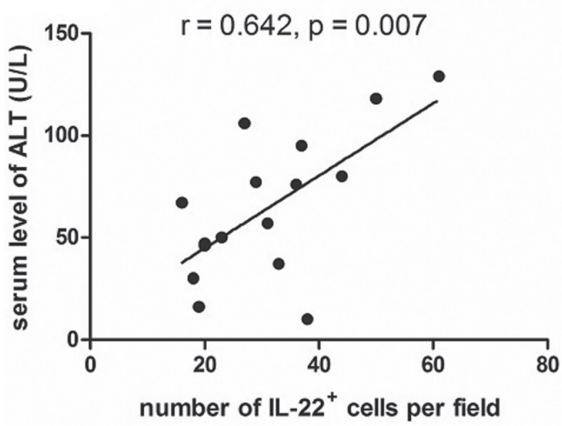

C

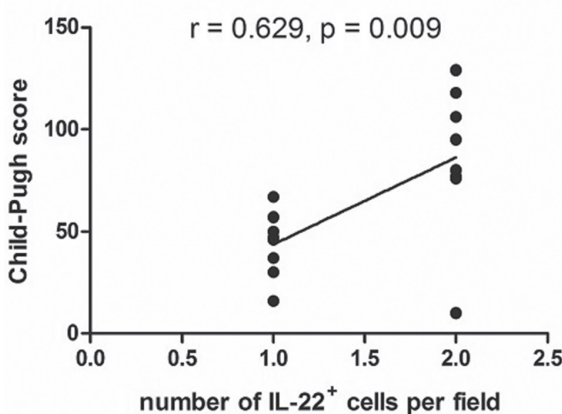

B

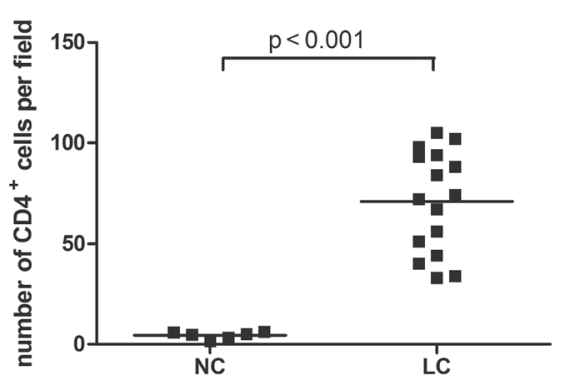

D

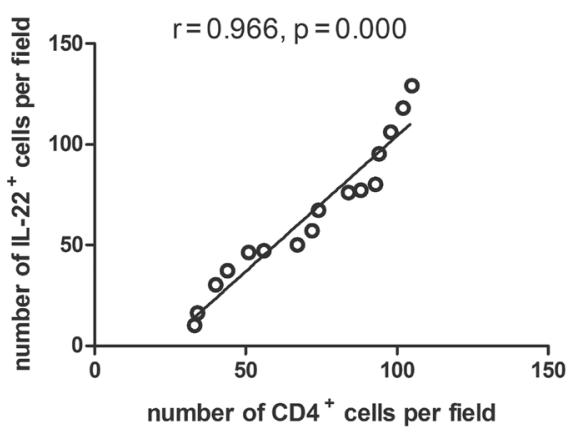

B

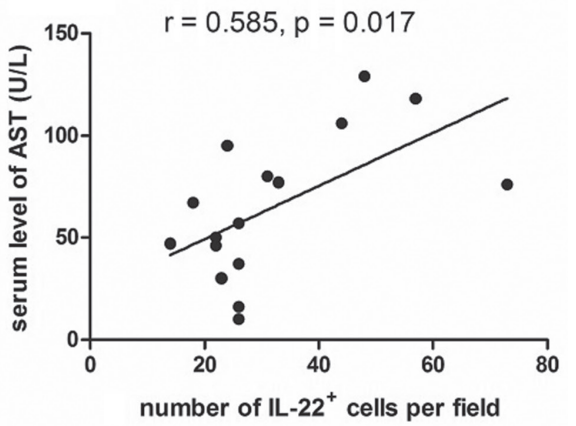

D

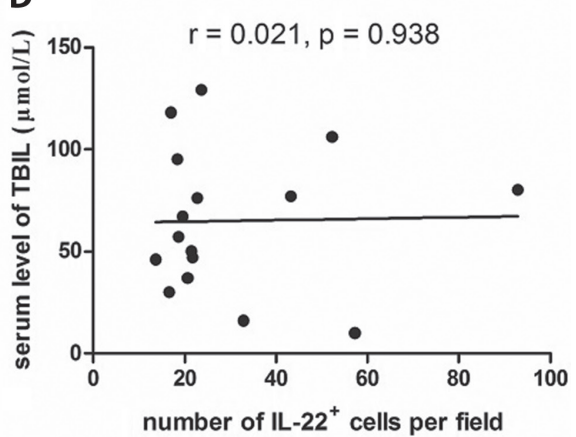

Fig. 2. Th22 cells and CD4+ cells accumulate in the livers of liver cirrhosis (LC) patients

(A, B) The number of Th22 and CD4 ${ }^{+}$ lymphocytes per field $(\times 400)$ in the liver of LC compared with normal controls (NC). The number of Th22 lymphocytes per field $(\times 400)$ in the liver is positively correlated with the number of $\mathrm{CD}^{+}$ lymphocytes in NC (C) and LC groups (D).
Fig. 3. Correlation between the number of Th22 cells and the clinical parameters in the liver cirrhosis (LC) group.

The number of Th22 cells was positively correlated with serum level of alanine aminotransferase (ALT) (A) and aspartate aminotransferase (AST) (B) and the ChildPugh score (C). There was no correlation between the number of Th22 cells and serum level of total bilirubin (TBIL) (D). $\mathrm{p}=0.007$, Fig. 3A), AST $(\mathrm{r}=0.585, \mathrm{p}=0.017$, Fig. 3B) and the Child-Pugh score $(r=0.629, p=0.009$, Fig. $3 C)$. There was no correlation between the number of Th22 cells and the serum level of TBIL ( $r=0.021, p=0.938$, Fig. 3D).

\section{Increased Th22 cell-related cytokines in the LC patients' livers}

Interleukin 22 is the effector cytokine of Th22 cells, and plays its role through a heterodimeric transmembrane receptor complex consisting of IL-10R2 and IL-22R1. 


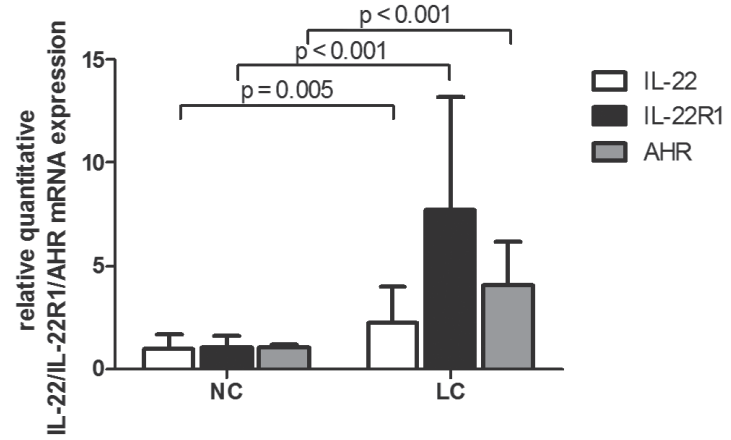

Fig. 4. Relative liver gene expressions of interleukin 22 (IL-22), IL-22R1 and aryl hydrocarbon receptor (AHR) were detected using real-time polymerase chain reaction (RT-PCR)

Because signaling through IL-22R1 is restricted to IL-22, we focused on IL-22 and IL-22R1. Aryl hydrocarbon receptor is the key transcription factor of Th22 cells, which control the production of IL-22. In the present study, we found that the amounts of IL-22, IL-22R1 and AHR mRNA were significantly increased in the LC patients as compared with the NC group ( $\mathrm{p}<0.05$, Fig. 4$)$.

\section{Discussion}

Liver cirrhosis is a chronic progressive disease characterized by the formation and accumulation of ECM and the activation of hepatic stellate cells (HSCs). ${ }^{10}$ Liver injury caused by HBV is strongly linked with cellular immunity and the immune tolerance of the organism. ${ }^{11} \mathrm{CD} 4^{+} \mathrm{T}$ cells participate in the immune response of the body and play a key role in the process of liver inflammation and fibrosis..$^{12}$ Although previous studies have found that Th22 cells play an important role in certain autoimmune diseases, such as gastric cancer, ${ }^{7}$ the mechanism of Th22 cells in HBV-associated LC has not been fully elucidated. In the present study, we confirmed that, like Th17 cells and Th1 cells, Th22 cells were present in liver injury, ${ }^{13}$ and that the number of Th22 cells and $C D 4{ }^{+} \mathrm{T}$ cells in LC was much higher than in the $\mathrm{NC}$ group. Moreover, we found that Th22 cells were correlated with $\mathrm{CD}^{+} \mathrm{T}$ cells in LC, suggesting that Th22 cells may be involved in the pathogenesis of HBV-associated LC.

As Th22 cells are abundantly expressed in cirrhotic liver tissues, to further understand their relationship with liver inflammation and fibrosis indices, we conducted a correlation analysis between the number of Th22 cells and the serum parameters of ALT, AST and TBIL, and the ChildPugh score. Our data showed that the number of Th22 cells was positively correlated with serum levels of ALT and AST and the Child-Pugh score, but the correlation with serum levels of TBIL did not reach statistical significance. These results were in line with findings of Park et al., who reported that the number of Th22 cells correlated positively with the serum levels of AST and ALT in patients with viral hepatitis. ${ }^{14}$ Taken together, these results indicate that
Th22 cells are associated with the severity of the disease and contribute to the prognosis.

The effector cytokine of Th22 cells is IL-22, which mediates its effects through IL-22R1 and IL-10R2. ${ }^{15,16}$ The former is mainly expressed at the highest levels in the pancreas, followed by the small intestine, colon, kidney, and liver. ${ }^{16}$ Kong et al. demonstrated that HSCs express high levels of IL-10R2 and IL-22R1. ${ }^{17}$ In the current study, we also found that the amount of IL-22 and IL-22R1 mRNA were significantly elevated in LC patients compared with the NC group. A high expression of IL-22 and IL-22R1 mRNA in liver tissue may effectively support signaling directionality from the immune system to the liver, and thus participate in the pathological process of LC.

Aryl hydrocarbon receptor is the major transcriptional factor of Th22 cells. ${ }^{4}$ CD $4{ }^{+} \mathrm{T}$ cells from AHR-deficient mice fail to produce IL-22 when exposed to AHR ligands, while they still develop normal Th17 cell responses. ${ }^{18}$ Trifari et al. also reported that transfection of AHR-specific siRNA into $C D 4^{+}$memory $T$ cells resulted in significantly lesser IL-22 production, but had no effect on IL-17; AHR agonists promote IL-22 production in humans. ${ }^{4}$ In accordance with previous studies, we observed that the expression of AHR in the LC patients was significantly increased in comparison with the control participants. We therefore deduced that overexpression of AHR induces Th22 cells to secrete more IL-22, which is closely related to Th22 cell function activities.

\section{Conclusions}

Our data showed that intrahepatic Th22 cells were significantly increased in LC patients and had a positive correlation with $\mathrm{CD} 4{ }^{+} \mathrm{T}$ cells. Additionally, overexpression of IL-22, IL-22R1 and AHR confirmed that Th22 cells may participate in the immunopathogenesis of HBV-associated LC. Th22 cells also contribute to the progression of liver fibrosis and can be used as a marker for the severity of LC.

\section{References}

1. Harrington LE, Hatton RD, Mangan PR, et al. Interleukin 17-producing CD4 ${ }^{+}$effector $T$ cells develop via a lineage distinct from the Thelper type 1 and 2 lineages. Nat Immunol. 2005;6(11):1123-1132.

2. Vierling JM. The immunology of hepatitis B. Clin Liver Dis. 2007;11(4): 727-759.

3. Duhen T, Geiger R, Jarrossay D, Lanzavecchia A, Sallusto F. Production of interleukin 22 but not interleukin 17 by a subset of human skin-homing memory T cells. Nat Immunol. 2009;10(8):857-863.

4. Trifari S, Kaplan CD, Tran EH, Crellin NK, Spits H. Identification of a human helper $\mathrm{T}$ cell population that has abundant production of interleukin 22 and is distinct from $\mathrm{T}(\mathrm{H})-17, \mathrm{~T}(\mathrm{H}) 1$ and $\mathrm{T}(\mathrm{H}) 2$ cells. Nat Immunol. 2009;10(8):864-871.

5. Kagami S, Rizzo HL, Lee JJ, Koguchi Y, Blauvelt A. Circulating Th17, Th22, and Th1 cells are increased in psoriasis. JInvest Dermatol. 2010; 130(5):1373-1383.

6. Brand S, Beigel F, Olszak T, et al. IL-22 is increased in active Crohn's disease and promotes pro-inflammatory gene expression and intestinal epithelial cell migration. Am J Physiol Gastrointest Liver Physiol. 2006;290(4):G827-838. 
7. Liu T, Peng L, Yu P, et al. Increased circulating Th22 and Th17 cells are associated with tumor progression and patient survival in human gastric cancer. J Clin Immunol. 2012;32(6):1332-1339.

8. Mookerjee RP, Stadlbauer V, Lidder S, et al. Neutrophil dysfunction in alcoholic hepatitis superimposed on cirrhosis is reversible and predicts the outcome. Hepatology. 2007;46(3):831-840.

9. Tritto G, Bechlis Z, Stadlbauer V, et al. Evidence of neutrophil functional defect despite inflammation in stable cirrhosis. J Hepatol. 2011; 55(3):574-581.

10. Friedman SL. Mechanisms of hepatic fibrogenesis. Gastroenterology. 2008;134(6):1655-1669.

11. Liaw Y-F, Chu C-M. Hepatitis B virus infection. Lancet. 2009;373(9663): 582-592.

12. Karlmark KR, Wasmuth HE, Trautwein C, Tacke F. Chemokine-directed immune cell infiltration in acute and chronic liver disease. Expert Rev Gastroenterol Hepatol. 2008;2(2):233-242.

13. Lai $R$, Xiang $X, M o R$, et al. Protective effect of Th22 cells and intrahepatic IL-22 in drug induced hepatocellular injury. J Hepatol. 2015;63(1): 148-155.
14. Park $\mathrm{O}$, Wang $\mathrm{H}$, Weng $\mathrm{H}$, et al. In vivo consequences of liver-specific interleukin-22 expression in mice: Implications for human liver disease progression. Hepatology. 2011;54(1):252-261.

15. Dumoutier L, Louahed J, Renauld JC. Cloning and characterization of IL-10-related T cell-derived inducible factor (IL-TIF), a novel cytokine structurally related to IL-10 and inducible by IL-9. J Immunol. 2000;164(44):1814-1819.

16. Kotenko SV, Izotova LS, Mirochnitchenko OV, et al. Identification of the functional interleukin-22 (IL-22) receptor complex: The IL-10R2 chain (IL-10Rbeta) is a common chain of both the IL-10 and IL-22 (IL10-related T cell-derived inducible factor, IL-TIF) receptor complexes. J Biol Chem. 2001;276(4):2725-2732.

17. Kong $X$, Feng $D$, Wang $H$, et al. Interleukin-22 induces hepatic stellate cell senescence and restricts liver fibrosis in mice. Hepatology. 2012;56(3):1150-1159.

18. Veldhoen $M$, Hirota $K$, Westendorf AM, et al. The aryl hydrocarbon receptor links TH17-cell-mediated autoimmunity to environmental toxins. Nature. 2008;453(7191):106-109. 